\title{
Média da Frequência Fundamental dos Formantes e do Tempo Máximo de Fonação em um Grupo de Escolares
}

\section{Mean of The Fundamental Frequency, of The Formants and the Maximum Phonation Time in a Group of Schoolchildren}

\author{
Raquel Elpídio Pinheiro da Silva ${ }^{1}$ \\ Lourdes Bernadete Rocha de Souza²
}

\section{RESUMO}

Objetivo: Determinar a média da frequência fundamental, dos formantes e o tempo máximo de fonação em um grupo de escolares. Metodologia: Foram selecionadas 35 meninas e 28 meninos, na faixa etária de 8 a 12 anos, estudantes do ensino fundamental e médio de uma escola em Natal/RN. O protocolo de gravação das vozes foi realizado a partir da emissão de vogais sustentadas e emissão da frase: Eu digo $/ a /, / \varepsilon /$, /i/, $/ \mathrm{J} / \mathrm{/u} /$, eu digo. Utilizou-se o software de análise acústica dos sons da fala PRAAT para a gravação e análise dos parâmetros. Resultados: Não ocorreu diferença estatisticamente significativa entre os grupos em relação à média de f0 e do TMF. Quanto à frequência dos formantes, os resultados mostraram diferenças estatisticamente significativas entre os sexos na maioria dos parâmetros, exceto nos valores de F3[a], F1[u], F2[u] e F3[u]. Conclusão: As medidas de tempo máximos de fonação e da f0, em relação ao sexo, não foram diferentes, enquanto, os formantes das vogais [a] e [i] demonstraram que ocorreram ajustes motores diferentes entre os sexos.

DESCRITORES

Criança. Voz. Fonação. Acústica.

\begin{abstract}
Objective: In view of the clinical value that the acoustic evaluation measures provide, of the few studies in the literature that standardize voice/speech characteristics of children without vocal complaints, we aimed to determine the average of the fundamental frequency, formants and maximum phonation time in a group of schoolchildren. Methodology: 35 girls and 28 boys were selected, aged 8 to 12 years, students of elementary and high school at a school in Natal/RN. The voice recording protocol was performed by issuing sustained vowels and issuing the sentence: I say /a/, / / /, /i/, /J/, /u/, I say. The acoustic analysis of speech sounds PRAAT was used for recording and analyzing the parameters. Results: There was no statistically significant difference between the groups in relation to the mean of $\mathrm{f} 0$ and TMF. As for the frequency of the formants, the results showed statistically significant differences between the sexes in most of the parameters except in the values of F3 [a], F1 [u], F2 [u] and F3 [u]. Conclusion: The measures of maximum phonation time and $\mathrm{f} 0$, in relation to sex, were not different. While the formants of the vowels [a] and [i] demonstrated that different motor adjustments occurred between the sexes.
\end{abstract}

\section{DESCRIPTORS}

Child. Voice. Phonation. Acoustics.

\footnotetext{
${ }^{1}$ Fonoaudióloga pela UFRN, residente no Programa de Saúde Auditiva do Hospital de Anomalias Craniofaciais da USP/Bauru. Bauru, São Paulo, Brasil.

${ }^{2}$ Fonoaudióloga, Professora Associada II do Departamento de Fonoaudiologia da Universidade Federal do Rio Grande do Norte. Natal, RN, Brasil.
} 
A avaliação vocal infantil deve considerar os aspectos adequados e inadequados da qualidade vocal. Para tanto, devem estar claros os parâmetros que caracterizam uma voz com e sem disfonia. A avaliação vocal deve contemplar a avaliação acústica da voz, a avaliação perceptivo-auditiva, a avaliação aerodinâmica e as avaliações do impacto psicossocial das perturbações vocais ${ }^{1}$.

A análise acústica, considerada como uma avaliação objetiva, é uma das ferramentas utilizadas para fins clínicos e de pesqui$\mathrm{sa}^{2,3}$. Favorecida pelos avanços tecnológicos envolve programas específicos de análise da $v_{0 z}^{4,5}$ e tem propiciado o surgimento de medidas fidedignas e de rápida obtenção ${ }^{6}$. Infere indiretamente os padrões vibratórios das pregas vocais e, ainda, a conformação do trato vocal ${ }^{6}$. Além disso, oferece informações quantitativas que permitem deduzir sobre a função laríngea, além de ser um instrumento não invasivo que facilita a sua utilização na pesquisa e na prática clínica ${ }^{7}$, valendo ressaltar que tal análise também auxilia no diagnóstico ${ }^{3}$ e no monitoramento do tratamento ${ }^{3,8}$.

No que diz respeito à conformação do trato vocal, os formantes são parâmetros da análise acústica, expressos por meio de seu valor médio em Hertz $(\mathrm{Hz})$, designados por $F_{1,} F_{2}, F_{3 . .} F_{n}$ de modo progressivo ${ }^{11}$. O primeiro formante $\left(\mathrm{F}_{1}\right)$ está relacionado à abertura da boca, o segundo $\left(F_{2}\right)$ tem relação direta com o deslocamento ântero-posterior da língua e o terceiro formante (F3) está relacionado com o tamanho da cavidade oral ${ }^{12,13}$.

A avaliação aerodinâmica contempla o tempo máximo de fonação (TMF) o qual é obtido pela média do tempo máximo que um indivíduo consegue sustentar uma vogal, numa só expiração, permitindo uma investigação quantitativa e qualitativa da fonação $0^{9,10}$.

Outra avaliação objetiva é a frequência fundamental (f0), que é um importante parâmetro na avaliação, tendo como objetivo avaliar a interação entre o comprimento, massa e tensão das pregas vocais durante a fonação, ou seja, a frequência da voz.

Diante do valor clínico que as medidas de avaliação acústica fornecem e, de poucos estudos na literatura que normatizam as características de voz/fala, em crianças sem queixas vocais, o presente estudo teve como objetivo verificar a média do TMF, da f0 e dos formantes em um grupo de escolares falantes do português brasileiro na Cidade de Natal.

\section{METODOLOGIA}

Este estudo foi aprovado pelo Comitê de Ética em Pesquisa do Hospital Universitário em Natal, sob número: CAAE 3504.0.000294-10. Apenas as informações quantitativas e qualitativas concernentes ao que propõe a pesquisa tiveram valor para fins de divulgação cientifica.

O desenho desta pesquisa foi do tipo transversal, observacional, realizado com um grupo de 63 crianças, de ambos os sexos, sendo 35 do sexo feminino e 28 do sexo masculino, com idade variando de 8 a 12 anos, com média de idade de 9,7 anos, estudantes do ensino fundamental e médio, residentes e falantes, nativos da Cidade de Natal. Foram excluídas as crianças com alguma alteração motora e/ou neurológica, que pudessem comprometer a fonação ao nível respiratório, fonatório e/ou respiratório. 
Para realizar a coleta, foi realizada a listagem de algumas escolas estaduais e municipais. A solicitação foi realizada pessoalmente pela pesquisadora aos diretores das escolas, que assinaram o termo de consentimento livre e esclarecido autorizado pelos pais das crianças, demonstrando ciência da confidencialidade do trabalho. Além disso, os participantes assinaram o termo de assentimento. Após a permissão, foi realizada a escolha das classes que continham alunos com os critérios estabelecidos para a pesquisa. Posteriormente, foi escolhido um local silencioso na escola em que fosse possível a realização da coleta.

Para coletar o tempo máximo de fonação, foi solicitado que cada criança realizasse uma inspiração com posterior emissão de cada vogal (/a/; /i/; /u/) de maneira prolongada e confortável. Para coletar o valor da frequência fundamental e dos formantes $\mathrm{F} 1$, F2 e F3 das vogais, foi solicitado de cada criança a emissão da frase: "Eu digo /a/, /ع/, /i/, /o/, /u/, eu digo", favorecendo, assim, uma emissão mais aproximada na fala coloquial e estendendo cada vogal por um tempo médio de 2 segundos. Para a gravação foi utilizado um microfone da marca Clone, posicionado a $5 \mathrm{~cm}$ da boca do voluntário, acoplado a um laptop HP. A f0 foi obtida a partir da seleção da emissão da vogal /a/ da frase e as frequências dos formantes F1, F2 e F3 foram obtidas a partir da seleção da região central de cada emissão da vogal da frase. A extração dos valores da f0 e dos formantes foi realizada por meio do software de análise acústica dos sons da fala PRAAT ${ }^{14}$ considerando a faixa de amostragem de $44.100 \mathrm{~Hz}, 16 \mathrm{Bit}$, mono canal.

Os resultados foram organizados em banco de dados no software estatístico PSPP 2.0. Para verificar o padrão de normalidade entre as variáveis foi utilizado o teste Kolmogorov-Smirnov. As variáveis que apresentaram distribuição normal foram submetidas ao teste $t$ de Student e as que não apresentaram distribuição normal, foi aplicado o teste de Mann-Whitney. A significância estatística considerada foi menor que $5 \%$.

\section{RESULTADOS}

A média e o desvio-padrão dos valores de f0 e TMF e da frequência dos formantes de ambos os sexos estão descritos nas Tabelas 1 e 2 , respectivamente.

Não existiu diferença estatisticamente significativa entre os grupos em relação à média de f0 e do TMF.

Quanto à frequência dos formantes, os resultados mostraram diferenças estatisticamente significativas entre os sexos na maioria dos parâmetros $(p<0,05)$, exceto nos valores de F3[a], F1[u], F2[u] e F3[u].

\section{DISCUSSÃO}

O presente estudo apresentou como objetivo verificar os valores da média do TMF, da f0 e dos três primeiros formantes de um grupo de escolares. Não ocorreu diferença estatisticamente significativa entre os dois grupos quanto à média da f0 (Tabela 1) e concorda com resultados de outro estudo que não encontrou diferenças significantes na média desse parâmetro entre os sexos ${ }^{15}$.

No entanto, o presente estudo revelou resultados discordantes, quando comparados com dados da literatura ${ }^{16}$, que apresenta- 
Tabela 1. Média e desvio padrão da frequência fundamental e tempo máximo de fonação das meninas e dos meninos

\begin{tabular}{|c|c|c|c|}
\hline $\begin{array}{c}\text { Variables } \\
(\mathrm{Hz})\end{array}$ & $\begin{array}{c}\text { meninas }(n=34) \\
\text { média }( \pm S D)\end{array}$ & $\begin{array}{c}\text { meninos }(n=28) \\
\text { média }( \pm S D)\end{array}$ & Valor-p \\
\hline F0 [a] & $226,46( \pm 19,91)$ & $227,70( \pm 17.42)$ & 0,79 \\
\hline MPT & & & \\
\hline [a] & $13,67( \pm 4,23)$ & $14,12( \pm 5,64)$ & 0,72 \\
\hline [i] & $13,25( \pm 4,13)$ & $13,86( \pm 4,76)$ & 0,59 \\
\hline [u] & $13,20( \pm 4,13)$ & $13,51( \pm 3,91)$ & 0,76 \\
\hline
\end{tabular}

F0: Frequência fundamental, MPT: Tempo máximo de fonação

$p<0.05$ Teste T de Student

Tabela 2. Média e desvio padrão dos três primeiros formantes das meninas e dos meninos

\begin{tabular}{c|c|c|c}
\hline $\begin{array}{c}\text { Variables } \\
(\mathrm{Hz})\end{array}$ & Meninas $(\mathrm{n}=34)$ & Meninos $(\mathrm{n}=28)$ & Valor-p \\
\hline F1[a] & $1050,61( \pm 106,94)$ & $969,87( \pm 103,45)$ & $0.007^{* *}$ \\
F2[a] & $719,80( \pm 72,84)$ & $704,31( \pm 87,94)$ & $0,006^{*}$ \\
F3[a] & $591,35( \pm 420,46)$ & $508,33( \pm 147,59)$ & $0,324^{*}$ \\
F1[i] & $1661,75( \pm 133,10)$ & $1578,18( \pm 86,97)$ & $0,006^{*}$ \\
F2[i] & $1818,66( \pm 294,74)$ & $1942,20( \pm 290,42)$ & $0,025^{* *}$ \\
F3[i] & $2756,02( \pm 227,64)$ & $2908,99( \pm 343,18)$ & $0,018^{*}$ \\
F1[u] & $529,30( \pm 146,49)$ & $508,33(147,59)$ & $0,371^{*}$ \\
F2[u] & $1662,84( \pm 400,17)$ & $1980,03( \pm 539,34)$ & $0,137^{* *}$ \\
F3[u] & $3078,37( \pm 293,92)$ & $3166,92( \pm 320,91)$ & $0,270^{*}$ \\
\hline F: Formantes. $p<0.05$ & $-{ }^{*}$ Teste T de Student; ** Mann-Whitney &
\end{tabular}

ram média da f0 mais aguda que o grupo do presente estudo. A justificativa para esta discordância pode estar relacionada com a metodologia do estudo, como também, guarda relação as características dos falantes, com ajustes motores distintos em diferentes regiões do país e de outras etnias.

O TMF é uma tarefa comum para a avaliação clínica dos componentes respiratórios e do mecanismo de fonação durante a produção da voz. A avaliação de vogais sustentadas revela a possibilidade de controle das forças aerodinâmicas da corrente pulmonar e mioelásticas da laringe. Por meio desta tarefa é possível avaliar a qualidade da emissão, uma vez que evidencia alterações como instabilidade, tremor, quebras de sonoridade, quebras de frequência e ruído da fonte glótica ${ }^{17-19}$.

De maneira geral, autores ${ }^{20}$ argumentam que as crianças que estão em idade escolar e que não possuem alterações vocais deveriam ser capazes de sustentar a emissão com um tempo aproximado de 10 segundos, resultado esse obtido com valores acima no presente estudo. 
A média do TMF foi ligeiramente maior no sexo masculino, no entanto, esta diferença não foi estatisticamente significativa e revela que as avaliações clínicas em crianças não devem levar em consideração as diferenças de sexo existentes para esse parâmetro.

Os formantes são harmônicos intensificados no trato vocal e espera-se que seus valores sejam mais agudos quanto maior for a f0. Apesar da f0 não ter apresentado diferenças entre os sexos neste estudo, as meninas revelaram $\mathrm{F} 1$ mais agudo na vogal [a] que os meninos e os resultados revelaram diferença estatisticamente significante entre os grupos. Esta diferença pode ser justificada, possivelmente, esteja relacionado ao ajuste motor, mostrando-se diferente entre os sexos, ou seja, as meninas apresentaram maior movimento de abertura da cavidade oral que os meninos, apresentando assim, valor de F1 da vogal [a] mais agudo.

\section{REFERÊNCIAS}

1. Hoffmann CF. Características vocais espectrográficas de crianças sem disfonia. Santa Maria: Universidade Federal de Santa Maria, 2019. Tese de Doutorado.

2. Soltani M, Ashayeri H, Modarresi $\mathrm{Y}$, Salavati M, Ghomashchi H.Fundamental frequency changes of Persian speakers across the life span. J Voice. 2014;28(3):274281.

3. Demirhan E, Unsal EM, Yilmaz C, Ertan E. Acoustic voice analysis of young turkish speakers. J Voice. 2016; 30(3):378. e21-378.e25.

4. Xue SN, Deliyski D. Effects of aging on selected acoustic voice parameters: preliminary normative data and educational implications. Educational gerontology. $2001 ; 27(2): 159-168$

5. Mendes AP, Ferreira LJL, Castro E. Softwares e hardwares de análise acústica da voz e da fala. Distúrb. Comun. 2012; 24(3):421-430.

6. Behlau M. Avaliação de voz. In: Behlau M (ed). Voz: o livro do especialista. São Paulo: Revinter; 2001. v.1. p.85-245.
Quanto aos formantes da vogal [i] os resultados foram estatisticamente significantes entre os sexos, revelando-se mais agudo nas meninas em $\mathrm{F} 1$ e mais agudo nos meninos em F2 e F3. O que justifica esta diferença reside no fato das meninas terem apresentado maior movimento articulatório da cavidade oral e terem aumentado este valor em $\mathrm{F} 1$ e os meninos realizado o ajuste motor com maior anteriorização da língua, aumentando o espaço faríngeo com maiores valores de F2 e F3.

\section{CONCLUSÃO}

Diante dos resultados obtidos concluiu-se no presente estudo que as medidas de tempo máximo de fonação e da fo, em relação ao sexo, não foram diferentes. Os formantes das vogais [a] e [i] demonstraram que ocorreram ajustes motores diferentes entre os sexos.

7. Spazzapan EA, Marino VCC, Cardoso VM, Berti LC, Fabron EMG. Características acústicas da voz em diferentes ciclos da vida: revisão integrativa da literatura. Rev. CEFAC. 2019; 21(3):e15018.

8. Goy H, Fernandes DN, Pichora-Fuller MK, van Lieshout P. Normative voice data for younger and older adults. J Voice. 2013; 27(5):545-555.

9. Behlau M, Feijo D, Madazio G, Rehder MI, Azevedo R Ferreira AE. Voz profissional: aspectos gerais e atuação fonoaudiológica. In: Behlau M, organizador. Voz: o livro do especialista. Vol 2. Rio de Janeiro: Revinter; 2005. P. 86-171.

10. Sundberg J. Ciência da voz: fatos sobre a voz na fala e no canto. São Paulo: Edusp; 2015.

11. Cukier S, Camargo Z. Abordagem da qualidade vocal em um falante com deficiência auditiva: aspectos acústicos relevantes do sinal de fala. Revista CEFAC. 2005;1(7):93-101. 
12. Behlau M, Azevedo R, Pontes P. Conceito de voz normal e classificação das disfonias. In: Behlau M, organizador. Voz - O Livro do especialista. 2a ed. Vol.1. Rio de Janeiro: Revinter; 2004.

13. Felipe AC, Grillo MH, Marotti M, Grechi TH. Normatização de medidas acústicas para vozes normais. Rev. Bras. Otorrinolaringol. 2006; 72(5):659-664.

14. Behlau M, Tosi O, Pontes P. Determinação da frequência fundamental e suas variações em altura ("jitter") e intensidade ("shimmer") para falantes do português brasileiro. Acta AWHO. 1985;4(1):5-10.

15. Navas DM. Análise computadorizada de freqüência fundamental e suas variaçöes em altura (jitter) e intensidade (shimmer) de vozes. Pró-Fono. 1989;1(1):22.

16. Tavares EL, Labio RB, Martins, RH. Estudo normativo dos parâmetros acústicos vocais de crianças de 4 a 12 anos de idade sem sintomas vocais: estudo piloto.Braz. J Otorhinolaryngol. 2010; 76(4):485-490.

17. Pinho SMR. Avaliação e tratamento de voz. In: Pinho SMR. Fundamentos em fonoaudiologia: tratando os distúrbios da voz. 2. ed. Rio de Janeiro: Guanabara Koogan; 2003. p. 3-40.
18. Gelfer MP, Bennet QE.Speaking fundamental frequency and vowel formant frequencies: effects on perception of gender. J of Voice. 2013; 27(5):556-566.

19. Aragão AN, Couto TE, Camargo ZA, Santos MAR, Gama ACC. Análise da qualidade vocal antes e após o uso profissional e social da voz. Audiology-Communication Research. 2014;19(3):209-214.

20. Prater DK, Swift RW. Manual of Voice therapy. Austin: Pro-ed; 1984.

\section{CORRESPONDÊNCIA}

Raquel Elpídio Pinheiro da Silva

Rua Albino Tambara 568, complemento: ao lado da USP Villa Universitária - Bauru - São Paulo CEP:17012470

Email: raquellelpi@outlook.com.br 